COUGH

\title{
Chronic cough: how do cough reflex sensitivity and subjective assessments correlate with objective cough counts during ambulatory monitoring?
}

\author{
Samantha Clare Decalmer, Deborah Webster, Angela Alice Kelsall, Kevin McGuinness, Ashley Arthur \\ Woodcock, Jaclyn Ann Smith
}

Thorax 2007;62:329-334. doi: 10.1136/thx.2006.067413

See end of article for authors' affiliations

\section{Correspondence to:}

Dr S C Decalmer, North West Lung Research Centre, Wythenshawe Hospital, Southmoor Road, Wythenshawe, Manchester M23 9LT, UK:

samdecalmer@hotmail.com

Received 28 June 2006

Accepted 9 October 2006

Published Online First

13 November 2006
Background: Cough reflex sensitivity, subjective estimates of cough frequency and cough-related quality of life have been used to assess cough and monitor treatment responses. The relationships between these measures and objective cough monitoring remain unclear and the usefulness of subjective assessments remains questionable.

Subjects: 62 patients with chronic cough (39 women) were studied. Mean age of patients was 54.9 (SD 12.2 ) years, with a median duration of cough of 5.5 (range 1-30) years.

Methods: Cough reflex sensitivity testing (C5; citric acid) was performed in all patients before fully ambulatory day-time and night-time cough recordings. Patients scored the frequency and severity of their cough (Visual Analogue Scales (VAS) and 0-5 score) for each recording period and completed a cough-related quality-oflife questionnaire, Leicester Cough Questionnaire (LCQ). Ambulatory cough recordings were manually counted and reported in terms of cough seconds per hour $(\mathrm{cs} / \mathrm{h})$. Cough rates were $\log _{10}$ transformed for analysis.

Results: The median time spent coughing was 11.36 (range 1.06-46) cs/h with median day rates of 15.59 (range $2-74.8$ ) cs/h and median night rates of 2.94 (range 0-26.67) cs/h. An inverse relationship was seen between day cough rates and $\log _{10} C 5(r=-0.452, p \leqslant 0.001)$. Subjective cough scores and visual analogue scales were only moderately associated with objective time spent coughing, with night-time being scores more strongly associated than day-time scores. The strongest correlation with objective cough frequency was cough-related quality of life (LCQ), $(r=-0.622, p \leqslant 0.001)$, mediated through the psychological domain. Conclusions: Subjective measures of cough and cough reflex sensitivity are only moderately related to objective time spent coughing, and hence cannot be used as surrogate markers for objective cough-frequency measurements. Cough-related quality of life (LCQ) is most strongly related to objectively counted cough, and may be a useful adjunct to objective measures in the assessment of cough.
C ough is the most common respiratory complaint for which medical attention is sought, and its impact is reflected in the $£ 93$ million spent on over-the-counter anti-tussives each year in the UK. ${ }^{1-3}$ Most coughs are acute and self-limiting, secondary to respiratory tract infection, but when cough becomes chronic ( $>8$-weeks duration) it can account for up to one third of all referrals to chest physicians. ${ }^{4}$

Chronic cough has a major effect on the quality of life, and patients consume substantial healthcare resources, often seeing several specialists over many years. It may prove diagnostically difficult, requiring multiple investigations and treatment trials, with diagnostic success quoted as ranging from $100 \%$ in a general respiratory clinic setting, ${ }^{5}$ to $58 \%$ in the specialist cough clinic setting. ${ }^{6}$

Assessment of cough and its response to treatment has mainly been based on subjective symptom perception using cough diary scores and cough Visual Analogue Scales (VAS). ${ }^{78}$ However, the lack of an objective gold standard in terms of objectively counted cough has meant that it has been impossible to assess the relative value of these different subjective measures. Cough reflex sensitivity testing has also been used as a surrogate index of disease severity, although it is not disease specific. ${ }^{9}$ The dose of an inhaled tussogenic substance such as citric acid or capsaicin (which usually induces 5 coughs in the minute after inhalation-C5) is reduced among patients with chronic cough and increases after successful treatment. ${ }^{10-12}$ However, whether improved C5 in an individual predicts an objective clinical response is unknown. A study of seven patients found a strong correlation with objective cough monitoring in adults with chronic cough when monitored for a short period $(6 \mathrm{~h})$ during the day, ${ }^{13}$ but whether this relationship holds for prolonged ambulatory, and especially home, monitoring is not known.

Cough-specific quality-of-life questionnaires have been developed, psychometrically tested and found to be reproducible and valid when compared with other quality-of-life measures. ${ }^{14} 15$ These have been used to monitor treatment responses, and the Leicester Cough Questionnaire (LCQ) correlates with short day-time recordings of objective cough counts. ${ }^{13}$ This relationship has not been explored with $24 \mathrm{~h}$ monitoring at home, where a quality-of-life measurement is most relevant.

An objective measure of cough would be valuable in the assessment of frequency, diurnal variation and treatment responses. The ideal cough monitor would be robust, unobtrusive and portable, and allow recording over at least $24 \mathrm{~h}$. It would be operator friendly, and able to identify all coughs, distinguishing them from all speech, sneezing, laughter and background noise, automatically. To date, an automated monitor has been used to study cough in chronic obstructive

Abbreviations: cs/h, cough seconds per hour; $L C Q$, Leicester Cough Questionnaire; Log C5, logarithmic transformation of concentration causing 5 coughs; VAS, Visual Analogue Scale 
Table 1 Cough scoring system adapted from $\mathrm{Hsu}$ et aP

\begin{tabular}{llll}
\hline Score & Daytime & Score & Night-time \\
\hline 0 & No cough during the day & 0 & No cough during the night \\
1 & Cough for one short period & 1 & Cough on waking only \\
2 & Cough for more than two short periods & 2 & Wake once or early due to cough \\
3 & Frequent coughing, which did not interfere with usual daytime activities & 3 & Frequent waking due to coughs \\
4 & Frequent coughing, which did interfere with usual daytime activities & 4 & Frequent coughs most of the night \\
5 & Distressing cough most of the day & 5 & Distressing cough most of the night \\
\hline
\end{tabular}

pulmonary disease in a controlled, non-ambulatory setting. This has been validated against video data, ${ }^{16}$ but sensitivity is low. Ambulatory monitors with manual counting have been used in patients with chronic cough, ${ }^{13}$ but only for short periods $(6 \mathrm{~h})$.

We have developed a method for fully ambulatory cough recording, validated against manual cough counts from video recordings, ${ }^{17-19}$ which is responsive to change. ${ }^{20}$ Currently, coughs are counted manually and quantified in terms of cough seconds per hour $(\mathrm{cs} / \mathrm{h})$, defined as the number of seconds containing at least one explosive cough sound,,$^{1721}$ with excellent interobserver and intraobserver agreement. ${ }^{17}{ }^{21}$

The aim of this study was to explore the relationships between subjective cough scores, cough quality of life, cough reflex sensitivity and objective time spent coughing derived from ambulatory home recordings in patients with chronic cough.

\section{PATIENTS AND METHODS}

Consecutive patients with chronic cough ( $>8$-weeks' duration), who were referred to the tertiary referral cough clinic at the North West Lung Centre, South Manchester University Hospitals Trust, Manchester, UK, were studied. Current smokers, ex smokers of <6-months' duration, those with a respiratory tract infection in the preceding month and those taking ACE inhibitors or opiates were excluded. A diagnosis of the underlying cause of chronic cough was established from the history, investigations and trials of treatment. Investigations in addition to those reported here included $24 \mathrm{~h} \mathrm{pH}$ monitoring, bronchoscopy, CT scanning and ENT examination. Diagnoses were established when resolution or considerable improvement of the cough occurred with specific treatment. Patients without definite findings on investigation and no response to treatment were considered idiopathic. All measures of cough were performed before investigation or trials of treatment.

The study was approved by the local research ethics committee and all patients gave written, informed consent. A sample size of 62 patients would have $80 \%$ power to detect correlation coefficients of $\geqslant 0.35$. In a previous study of patients with chronic obstructive pulmonary disease, ${ }^{22}$ we found that

Table 2 Summary statistics for objective and subjective measures of cough during the day and night

\begin{tabular}{lcll}
\hline & Day & Night & p Value \\
\hline Cough rate (cs/h) & $15.59(2-74.8)^{*}$ & $2.94(0-26.7)^{*}$ & $<0.001 \dagger$ \\
Cough score & $2.81(0.85) \ddagger$ & $1(0-4)^{*}$ & $<0.001 \dagger$ \\
Cough VAS (mm) & $40.6(23.7) \ddagger$ & $18(0-87)^{*}$ & $<0.001 \dagger$ \\
C5 (M) & $0.25(0.03-4)^{*}$ & NA & NA \\
Log C5 (M) & $-0.52(0.58) \dagger$ & NA & NA \\
\hline
\end{tabular}

$\mathrm{cs} / \mathrm{h}$, cough seconds per hour; $\log \mathrm{C} 5$, logarithmic transformation of concentration causing 5 coughs; VAS, Visual Analogue Scale. "Median (range).

+Wilcoxon signed rank test.

$\ddagger$ Mean (SD). the weakest correlation was between objective cough rate and cough scores $(0.37)$, hence we powered this study to detect correlations of this order and above.

\section{Cough reflex sensitivity}

A citric acid cough challenge test was conducted to assess the sensitivity of the cough reflex..$^{23}$ Six ascending concentrations (doubling doses from 0.03 to $1 \mathrm{M}$ ) were delivered as $12 \mu \mathrm{l}$ single-breath inhalations (Mefar Dosimeter, Brescia, Italy, or KoKo Dosimeter, De Villbis Health Care Inc, Somerset, Pennsylvania, USA, Pds Instrumentation, USA). Three placebo inhalations of normal saline were randomly interspersed, with both the operator and the patient blinded to their position. After each inhalation, the number of coughs in the subsequent minute was counted by an experienced observer. The challenge was terminated at the concentration of citric acid which resulted in 5 consecutive coughs (C5).

\section{Objective time spent coughing}

Ambulatory cough recording was started immediately after the cough challenge was completed; we have previously found that cough reflex testing does not markedly alter objective cough frequency in the subsequent $24 \mathrm{~h} .{ }^{24}$ Patients were discharged from hospital with the cough monitor in place, and instructed to return to their normal daily routine. Digital sound recordings were made during the day and overnight, as described previously ( $16 \mathrm{kHz}, 16$ bit wave format). ${ }^{182} 22$ Cough recordings were transferred from the digital recorders to a personal computer and archived on a compact disc. For each recording, coughs were counted manually in "cough seconds" - that is, the number of seconds per hour in which at least one explosive cough sound was present $(\mathrm{cs} / \mathrm{h}){ }^{21}$ If coughs occurred as a "peel", that is several cough sounds occurring after one inspiration, the total number of seconds in which these sounds were heard was recorded. We present objective cough data as time spent coughing per hour ( $\mathrm{cs} / \mathrm{h}$ ) for which we have shown a high level of agreement between observers. ${ }^{17}$

Cough monitoring was repeated in 10 patients after 14 days, to measure the repeatability of cough seconds.

\section{Subjective measures of cough}

Patients were asked to subjectively assess their cough severity for the day and night recording periods by means of a VAS ( $100 \mathrm{~mm}$ linear scale marked with a horizontal line by the patient, with $0 \mathrm{~mm}$ representing "no cough" and $100 \mathrm{~mm}$ "worst cough"). Subjective scores of cough frequency were measured by a cough scoring system completed by patients (table 1$){ }^{7}$

\section{Cough-related quality of life}

All patients completed the LCQ, a cough-specific quality-of-life measure. This is a 19-item validated, repeatable and responsive questionnaire, which contains physical, social and psychological domains. Answers are graded on a 7-point Likert scale, 
giving a total score ranging from 3 to 21 , a higher score indicating a better health status. ${ }^{14}$

\section{Statistical analysis}

Day, night and total cough rates were positively skewed; logarithmic transformation normalised the distribution of total and daytime rates, but not night-time rates. Night-time VAS and night-time cough scores were also positively skewed and were not normalised by logarithmic transformation. All other variables were normally distributed.

To examine correlations between objective and subjective measures, the appropriate parametric and non-parametric tests were performed using SPSS V.11.0. The relationships between the domains of the LCQ and objective cough rates were examined using a multiple linear regression analysis.

\section{RESULTS}

\section{Patients}

From 111 patients assessed in the cough clinic, 79 met the criteria for inclusion into the study. Of these, 66 agreed to participate in the study, but 4 patients failed to complete. Data are presented on 62 patients (39 women, 62.9\%) with a mean age of 54.9 years (standard deviation (SD) 12.2), mean forced expiratory volume in $1 \mathrm{~s} 101.8 \%$ (16.2\%) of predicted and mean FVC $107 \%(15.5 \%)$ of predicted. The median duration of coughing was 5.5 (range 1-30) years. At initial presentation to clinic, $29 \%(n=18)$ of patients were taking inhaled steroids, $1.6 \%(\mathrm{n}=1)$ oral steroids, $1.6 \%(\mathrm{n}=1)$ nasal steroids, $9.7 \%$ $(\mathrm{n}=6)$ antihistamines and $35.5 \% \quad(\mathrm{n}=22)$ proton pump inhibitors. In all, 23 patients were ex smokers, with a median period of abstinence of 15 (range 3-35) years.

Based on history, investigation and response to treatment trials, diagnosis was established in $64.5 \%(\mathrm{n}=40)$ of patients, and the remainder were classified as idiopathic $(n=22)$. One diagnosis was identified in $74.2 \%$ of patients, two in $21 \%$ and three in $4.8 \%$. The most common diagnosis was gastrooesophageal reflux disease $(n=33)$, followed by postnasal drip $(\mathrm{n}=8)$, asthma $(\mathrm{n}=6)$, eosinophilic bronchitis $(\mathrm{n}=2)$, bronchiectasis $(\mathrm{n}=2)$ and tracheopathia osteochondroplastica $(\mathrm{n}=2)$. Isolated cases of tracheomalacia, extrinsic allergic alveolitis, endobronchial amyloidosis, emphysema and respiratory bronchiolitis were also identified.

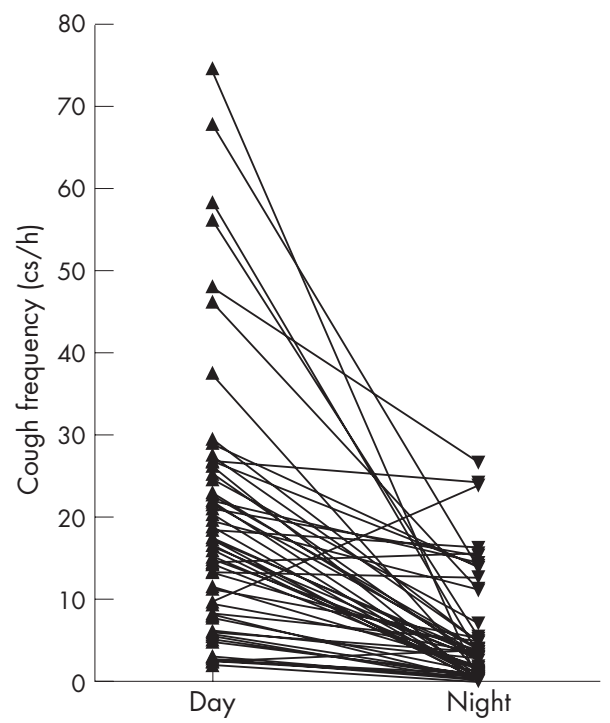

Figure 1 Time spent coughing during the day and night in individuals with chronic dry cough.

\section{Objective cough monitoring}

All 62 patients had successful daytime recordings, with 58 having successful overnight recordings (4 patients were unable to initiate overnight recordings despite repeated attempts). The median total time spent coughing was $11.36 \mathrm{cs} / \mathrm{h}$, with a wide range noted $(1.06-46 \mathrm{cs} / \mathrm{h})$. Median daytime cough rates of 15.59 (range 2-74.8) cs/h were substantially higher than median night-time rates of 2.94 (range $0-26.67$ ) cs/h; (Wilcoxon signed rank test, $\mathrm{p} \leqslant 0.001$; table 2 and fig 1 ).

The repeatability of cough monitoring was excellent. Daytime cough rates were similar for both visits (mean (range) days 1 and 14: $13.3(2-22.8) \mathrm{cs} / \mathrm{h}$ and $12(0.9-27) \mathrm{cs} / \mathrm{h}$, respectively; $\mathrm{p}=0.56)$, with a mean difference of $1.24 \mathrm{cs} / \mathrm{h}(95 \%$ confidence interval (CI) -11.3 to $13.8 \mathrm{cs} / \mathrm{h}$ ). Overnight cough rates were also reproducible between visits (mean (range) days 1 and 14: $3.7(0-17.2) \mathrm{cs} / \mathrm{h}$ and $2.67(0.5 \mathrm{l}-12.64) \mathrm{cs} / \mathrm{h}$, respectively; $\mathrm{p}=0.14)$, with a mean difference of $1.91 \mathrm{cs} / \mathrm{h}(95 \%$ CI -5.40 to $9.21 \mathrm{cs} / \mathrm{h})$.

Relationship between objective cough monitoring and cough reflex sensitivity

One patient coughed on a placebo inhalation of normal saline and therefore did not achieve a measurable C5. In the remaining 61 patients, the median concentration of citric acid inducing 5 coughs was 0.25 (range 0.03-4) M. As cough reflex sensitivity was measured during the day, we investigated relationships with daytime cough counts only. There was a significant inverse correlation between $\log _{10}$ daytime cough rates and $\log \mathrm{C} 5$ (Pearson's $\mathrm{r}=-0.45, \mathrm{p} \leqslant 0.001$; fig 2 ).

\section{Relationship between objective and subjective cough measures}

Subjective cough measures tended to correlate more strongly with objective cough rates for overnight than for daytime recordings and the VAS correlated more strongly than cough scores (table 3 ).

\section{Cough score}

Mean daytime scores were 2.82 (SD 0.85) and median nighttime scores 1 (range 0-4). Day and night cough scores showed moderate positive correlation with logarithmically transformed objective time spent coughing during the day and night, respectively (Pearson's, day $r=0.50, p \leqslant 0.001$ and Spearman's night $\mathrm{r}=0.55, \mathrm{p} \leqslant 0.001$ ).

\section{VAS scores}

A mean daytime VAS of 40.5 (SD 23.7) and median night-time VAS of 18 (range $0-87$ ) was seen. These positively correlated with $\log _{10}$ day and night cough rates, respectively (Pearson's, day $r=0.46, p \leqslant 0.001$, Spearman's night $r=0.61, p \leqslant 0.001$; fig 3).

\section{Cough-related quality of life (LCQ)}

All 62 patients completed the LCQ. The median total LCQ Score was 12.8 (SD 3.7); scores for the physical, psychological and social domains were 4.4 (SD 1.1), 4.3 (SD 1.5) and 4.1 (SD 1.5), respectively. A moderately strong negative correlation was seen between $\log _{10}$ total cough rates and total LCQ Score (Pearson's $\mathrm{r}=-0.62, \mathrm{p}=<0.001$; fig 4 and table 3 ). Similar correlations were seen in the physical, psychological and social domains when examined separately (table 3 ).

As the LCQ correlated most strongly with total objective cough frequency, a multiple linear regression analysis was used to examine the relative contribution of the subdomains to this relationship. In this analysis, only the psychological domain remained significantly related to objective cough frequency 


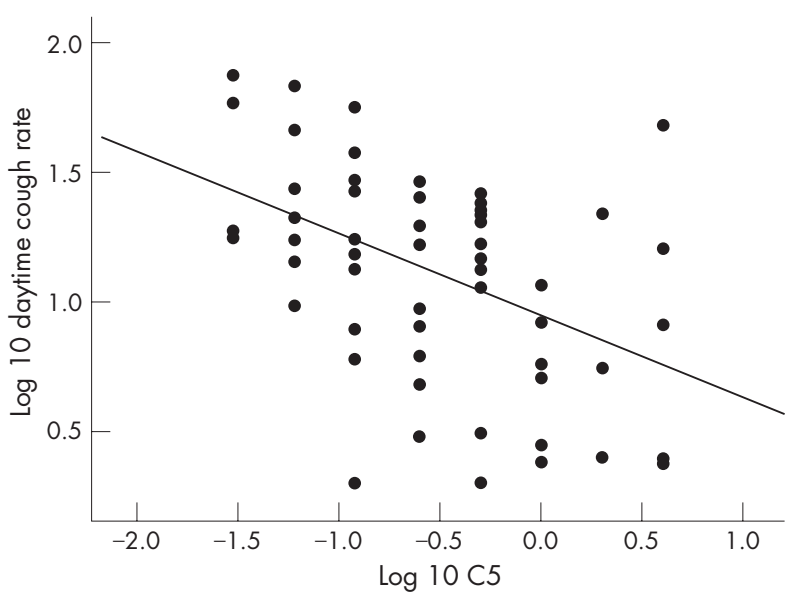

Figure 2 Correlation between cough reflex sensitivity and daytime cough rates.

$(\mathrm{B}=-0.13(95 \% \mathrm{CI}-0.19$ to -0.07$) ; \mathrm{p} \leqslant 0.001$, adjusted for age and sex).

The LCQ also correlated with both day and night cough frequency separately (table 3 ). Regression analysis for daytime, objective cough frequency found that the psychological subdomain, again, was significantly associated $(\mathrm{B}=-0.12$ (95\% CI -0.20 to -0.03 ); $\mathrm{p} \leqslant 0.001$ ). For overnight cough frequency, however, the physical domain alone was significant $(\mathrm{B}=-0.38$ (95\% CI -0.57 to -0.20$) ; \mathrm{p} \leqslant 0.001)$. Both analyses were also adjusted for age and sex.

\section{DISCUSSION}

This is the first study to examine the relationships between subjective measures of cough severity, cough-related quality of life, cough reflex sensitivity and objective cough rates monitored over $24 \mathrm{~h}$ in fully ambulatory adults with chronic cough.
Most previous studies have used either only subjective measures of cough, or cough-related quality-of-life questionnaires to assess the frequency and severity of cough, and monitor treatment responses. However, it has been impossible to assess their validity in the absence of an objective measure of cough frequency.

The use of cough seconds as a measure of cough frequency is different from other investigators who report cough sounds. However, a strong linear relationship has been shown between cough sounds and cough seconds, and excellent interobserver agreement has been noted, making this a comparable measure, giving an indication of overall time spent coughing. ${ }^{17}$

Cough rates measured in the home environment, with ambulatory monitoring over a 24 -h period, seem lower than those reported previously in the shorter studies in fewer patients, mainly under hospital surveillance. For example, Birring $\mathrm{et}^{\mathrm{al}} \mathrm{l}^{13}$ recorded mean cough rates of $43 \mathrm{cs} / \mathrm{h}$ in studies over $6 \mathrm{~h}$. There are a number of possible explanations for the discrepancy; firstly, the methods for quantifying cough are different. Cough seconds will always be less than the number of explosive cough phases. ${ }^{17}$ Secondly, it is our experience and that of others that cough rate data are positively skewed..$^{22}{ }^{25}$ The scatter plot of Birring et al's data suggests that these data may also be positively skewed and hence the mean would be an inappropriate summary statistic; the median appears to be about 17-that is, in agreement with our data. Finally, the patients were only monitored for a short period of time $(6 \mathrm{~h})$ and it is possible that the patients paid more attention to their urge to cough as they were aware of the cough monitor and hence coughed more. Over longer time periods, patients are more likely to be distracted and less influenced by the presence of the monitor.

Both cough scores and VAS showed a moderate correlation with cough frequency. The relationship between overnight cough frequency and subjective measures seems to be stronger than for daytime data, in contrast with a previous small study suggesting that night-time cough scores and observed rates

\begin{tabular}{|c|c|c|c|}
\hline & $\begin{array}{l}\text { Log total time spent } \\
\text { coughing }\end{array}$ & $\begin{array}{l}\text { Log daytime spent } \\
\text { coughing }\end{array}$ & $\begin{array}{l}\text { Log night-time spent } \\
\text { coughing }\end{array}$ \\
\hline \multicolumn{4}{|l|}{ Cough score } \\
\hline Day & NA & $\begin{array}{l}r=0.50 \\
p \leqslant 0.001\end{array}$ & NA \\
\hline Night & NA & NA & $\begin{array}{l}r=0.55 \\
p \leqslant 0.001^{*}\end{array}$ \\
\hline \multicolumn{4}{|l|}{ VAS } \\
\hline Day & NA & $\begin{array}{l}r=0.46 \\
p \leqslant 0.001\end{array}$ & NA \\
\hline Night & NA & NA & $\begin{array}{l}r=0.61^{*} \\
p \leqslant 0.001\end{array}$ \\
\hline \multicolumn{4}{|l|}{$\log _{10}$ C5 } \\
\hline Day & NA & $\begin{array}{l}r=-0.45 \\
p \leqslant 0.001\end{array}$ & NA \\
\hline \multicolumn{4}{|l|}{ LCQ } \\
\hline Total & $\begin{array}{l}r=-0.62 \\
p \leqslant 0.001\end{array}$ & $\begin{array}{l}r=-0.54 \\
p \leqslant 0.001\end{array}$ & $\begin{array}{l}r=-0.39 \\
p=0.002\end{array}$ \\
\hline Physical & $\begin{array}{l}r=-0.55 \\
p \leqslant 0.001\end{array}$ & $\begin{array}{l}r=-0.43 \\
p=0.001\end{array}$ & $\begin{array}{l}r=-0.53 \\
p \leqslant 0.001\end{array}$ \\
\hline Psychological & $r=-0.59$ & $r=-0.54$ & $r=-0.33$ \\
\hline & $p \leqslant 0.001$ & $P \leqslant 0.001$ & $p=0.012$ \\
\hline Social & $\begin{array}{l}r=-0.55 \\
p \leqslant 0.001\end{array}$ & $\begin{array}{l}r=-0.49 \\
p \leqslant 0.001\end{array}$ & $\begin{array}{l}r=-0.26 \\
p=0.053\end{array}$ \\
\hline
\end{tabular}



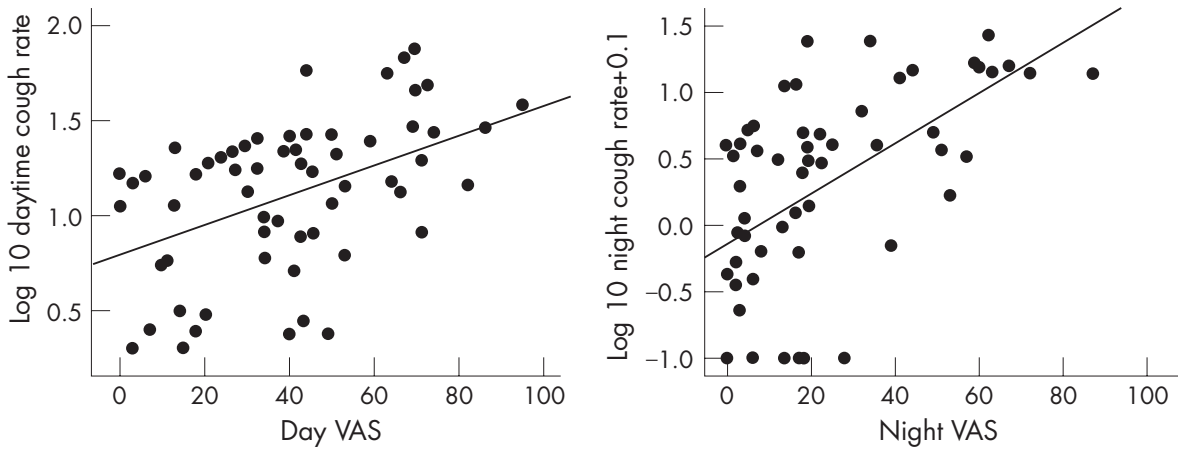

Figure 3 Correlation between objective cough frequency and Visual Analogue Scale (VAS). failed to correlate. ${ }^{7}$ We speculate that the stronger correlations, seen in our larger dataset, between objective night-time cough rates and subjective evaluation may result from sleep disturbance and fatigue. This interpretation tends to be supported by the cough quality-of-life data, which suggested that nocturnal coughing was independently related to cough-related quality of life, in particular the physical domain.

A retrospective analysis suggested that the strongest relationship existed with the psychological domain of the LCQ. In a recent study, $>50 \%$ of patients attending a specialist chronic cough clinic met the criteria on the Center for Epidemiologic Studies-Depression Scale for clinical depression. Furthermore, depression scores dramatically improved in patients whose cough was successfully treated. ${ }^{26}$ Psychological problems may increase the cough frequency, but it would seem more plausible that the patients with the highest cough rates have the greatest psychological consequences.

Data on the relationship between cough reflex sensitivity and cough frequency are limited. ${ }^{132527}$ All of the patients in this study had a measurable cough reflex sensitivity to citric acid. However, many normal volunteers also have a measurable cough reflex sensitivity in the absence of symptoms. We found only a moderate inverse correlation for the sensitivity of the cough reflex ( $\log _{10}$ C5) with observed cough frequency, suggesting that higher cough rates are only moderately associated with a more sensitive cough reflex. ${ }^{13}$ Cough reflex sensitivity testing is probably of utmost use in gaining insights into the mechanisms by which coughing occurs and treatments have their action, rather than in assessing severity of cough or response to treatment.

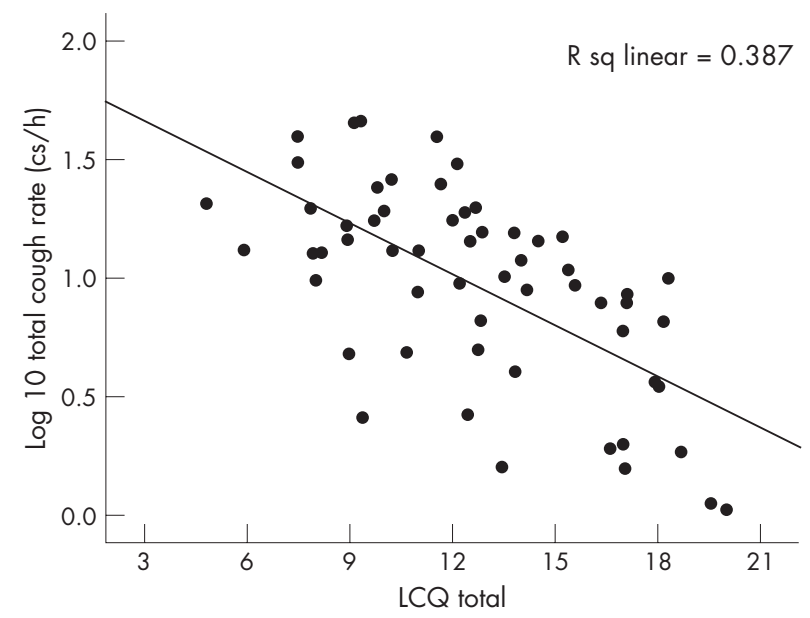

Figure 4 Correlation between total cough rates and total Leicester Cough Questionnaire (LCQ) Score.
Manually counting coughs from ambulatory recordings remains extremely laborious. Despite this, we found excellent interobserver and intraobserver agreement, ${ }^{17}$ and excellent agreement between cough rates over a 2-week period. Customised computer software is only now becoming available to allow automated counting of cough. This has proved a technical challenge, especially for daytime recordings where extraneous noise, particularly speech and background coughs may provide false positives. ${ }^{16}$ All recordings used in this study were manually counted in their entirety.

Although the assessment of objective cough rates represents a pronounced improvement in quantifying coughing, frequency may not be the only parameter that is important in a patient's perception of the severity of cough and its effect on quality of life. The intensity or effort of each cough may be important particularly in predicting the physical consequences of coughing. This should be possible in the future and it will be interesting to see whether cough intensity accounts for the remaining variance in subjective measures of cough not explained by cough frequency.

In summary, subjective measures of cough, cough-related quality of life and cough reflex sensitivity correlate modestly with objective measures of cough frequency in patients with chronic cough. Cough frequency seems to relate best to the psychological subdomain of cough-related quality of life rather than to social or physical subdomains. We suggest that, currently, a combination of objective cough frequency and cough-related quality of life is the optimal combination for assessing cough.

\section{ACKNOWLEDGEMENTS}

We thank all the patients who took part in the study. We also thank Dr Surinder Birring for his permission to use the Leicester Cough Questionnaire (LCQ) and the Moulton Charitable Trust for their financial support.

\section{Authors' affiliations}

Samantha Clare Decalmer, Deborah Webster, Angela Alice Kelsall, Kevin McGuinness, Ashley Arthur Woodcock, Jaclyn Ann Smith, University of Manchester, North West Lung Centre, South Manchester University Hospitals Trust, Manchester, UK

Funding: This study was funded by the North West Lung Centre Research Fund and Moulton Charitable Trust.

Competing interests: None.

\section{REFERENCES}

1 UK OTC Market Summary, 2004. (accessed 21 Dec 2006)

2 Schappert SM, Burt CW. Ambulatory care visits to physician offices, hospital outpatient departments, and emergency departments: United States, 2001-02. Vital Health Stat 13, 2006;159:1-66. 
3 Fuller RW, Jackson DM. Physiology and treatment of cough. Thorax 1990;45:425-30.

4 McGarvey LP, Heaney LG, MacMahon J. A retrospective survey of diagnosis and management of patients presenting with chronic cough to a general chest clinic. Int J Clin Pract 1998;52:158-61.

5 Irwin RS, Corrao WM, Pratter MR. Chronic persistent cough in the adult: the spectrum and frequency of causes and successful outcome of specific therapy. Am Rev Respir Dis 1981;123(Part 1):413-17.

6 Haque RA, Usmani OS, Barnes PJ. Chronic idiopathic cough: a discrete clinical entity? Chest 2005;127:1710-13.

7 Hsu JY, Stone RA, Logan-Sinclair RB, et al. Coughing frequency in patients with persistent cough: assessment using a 24 hour ambulatory recorder. Eur Respir J 1994;7:1246-53

8 Irwin RS, French CT, Smyrnios NA, et al. Interpretation of positive results of a methacholine inhalation challenge and 1 week of inhaled bronchodilator use in diagnosing and treating cough-variant asthma. Arch Intern Med 1997; 157:1981-7.

9 Morice AH, Fontana GA, Sovijarvi AR, et al. The diagnosis and management of chronic cough. Eur Respir J 2004;24:481-92.

10 Niimi A, Torrego A, Nicholson AG, et al. Nature of airway inflammation and remodeling in chronic cough. J Allergy Clin Immunol 2005; 116:565-70.

11 O'Connell F, Thomas VE, Pride NB, et al. Capsaicin cough sensitivity decreases with successful treatment of chronic cough. Am J Respir Crit Care Med 1994; 150:374-80.

12 McGarvey LP, Heaney LG, Lawson JT, et al. Evaluation and outcome of patients with chronic non-productive cough using a comprehensive diagnostic protocol. Thorax 1998;53:738-43.

13 Birring SS, Matos S, Patel RB, et al. Cough frequency, cough sensitivity and health status in patients with chronic cough. Respir Med 2006;100:1 105-9.

14 Birring SS, Prudon B, Carr AJ, et al. Development of a symptom specific health status measure for patients with chronic cough: Leicester Cough Questionnaire (LCQ). Thorax 2003;58:339-43.
15 French CT, Irwin RS, Fletcher KE, et al. Evaluation of a cough-specific quality-oflife questionnaire. Chest 2002;121:1123-31.

16 Coyle MA, Keenan DB, Henderson LS, et al. Evaluation of an ambulatory system for the quantification of cough frequency in patients with chronic obstructive pulmonary disease. Cough 2005;1:3

17 Smith J. The objective measurement of cough [PhD thesis]. Manchester: University of Manchester, 2004.

18 Smith J, Owen E, Earis J, et al. Effect of codeine on objective measurement of cough in chronic obstructive pulmonary disease. J Allergy Clin Immunol 2006; 117:831-5.

19 Smith JA, Earis JE, Woodcock A. Establishing a gold standard for manual cough counting: video versus digital audio recordings. Cough 2006;2:6.

20 Smith JA, Owen EC, Jones AM, et al. Objective measurement of cough during pulmonary exacerbations in adults with cystic fibrosis. Thorax 2006;61:425-9.

21 Smith J, Hiew Y, Cheetham B, et al. Cough seconds-a new measure of cough [abstract]. Am Thor Soc Conf May 2002:D99.

22 Smith J, Owen E, Earis J, et al. Cough in chronic obstructive pulmonary disease: correlation with cough challenge and subjective assessment. Chest 2006;130:379-85.

23 Wong $\mathrm{CH}$, Morice $\mathrm{AH}$. Cough threshold in patients with chronic obstructive pulmonary disease. Thorax 1999;54:62-4.

24 Webster D. The objective monitoring of cough in chronic cough patients [Mphil thesis]. Manchester: University of Manchester, 2005.

25 Chang $A B$, Phelan PD, Robertson CF, et al. Relation between measurements of cough severity. Arch Dis Child 2003;88:57-60.

26 Dicpingaitis $\mathbf{P}$, Tso R, Banauch $\mathrm{G}$. Prevalence of depressive symptoms amongst patients with chronic cough. Chest 2006;30:1839-43.

27 Webster D, Smith J, Hambleton E, et al. Does cough reflex sensitivity predict cough frequency in chronic cough?[abstract]. Am Thor Soc Conf 2005:A323. 